\title{
Unusual Balmer-Line Variations in the Radio-Loud AGN 4C 37.43
}

P. Romano and J. Sulentic

Department of Physics and Astronomy, University of Alabama, Tuscaloosa, AL 35487, USA

P. Marziani and M. Calvani

Osservatorio Astronomico, I-35122 Padova, Italy

M. Moles

Observatorio Astronomico Nacional, Madrid, Spain

T. Zwitter

Faculty of Mathematics and Physics, University of Ljubljana, Slovenia

\begin{abstract}
We present new observations of the quasar 4C 37.43 in the course of a more general study of broad-line profiles in radio-loud AGN. During the most recent observations it became obvious that the broad profile $\mathrm{H} \beta$ had changed significantly. Difference spectra show that a new blue component has appeared on the $\mathrm{H} \beta$ profile. The centroid of this new component is displaced about $2500 \mathrm{~km} \mathrm{~s}^{-1}$ from the rest frame [O III] $\lambda 5007$. A corresponding blueshifted He II $\lambda 4686$ component may have also appeared.
\end{abstract}

The 'classical double' radio source $4 \mathrm{C} 37.43$ is a low-redshift $(z=0.371)$, bright AGN $\left(M_{V}=-25.93 \mathrm{mag}\right)$. The optical observations are listed in Table 1 . Line profiles and difference spectra are shown in Fig. 1 (we thank P.T. O'Brien, M. Eracleous and T. A. Boroson for providing us access to their spectra).

Table 1. Observations

\begin{tabular}{llcccccc}
\hline \hline \multicolumn{1}{c}{ Dates } & Telescope & Spectr. & $\begin{array}{c}\text { Exp. } \\
(\mathbf{s})\end{array}$ & $\begin{array}{c}\text { Slit } \\
\left({ }^{\prime \prime}\right)\end{array}$ & $\begin{array}{c}\text { P.A. } \\
\left({ }^{\circ}\right)\end{array}$ & $\begin{array}{c}\text { Res. } \\
(\mathrm{A})\end{array}$ & Ref. \\
\hline May 5 1988 & La Palma 2.5-m & IDS & 8000 & 1.6 & & 3 & JPP91 \\
Sep. 20 1990 & KPNO 2.1-m & Gold & 2700 & 1.5 & 0 & $6.5-7$ & BG92 \\
Jul. 8 1992 & KPNO 4.0-m & & 1200 & 1.7 & 90 & 6 & EH94 \\
Apr. 11 1994 & Calar Alto 2.2-m & B\&C & 4800 & 2.0 & 90 & $5.6-8$ & M96 \\
May 1 1995 & Calar Alto 2.2-m & B\&C & 5400 & 1.6 & 90 & 6 & this work \\
\hline
\end{tabular}

The $\mathrm{H} \beta$ profile of $4 \mathrm{C} 37.43$ showed a relatively sudden change in the last $2-3$ years which represents an increase of $\sim 10 \%$ in the flux of the blue side in the rest frame. The change is concentrated in a displaced peak $\left(F W H M \approx 3000 \mathrm{~km} \mathrm{~s}^{-1}\right)$ shifted towards the blue by about $2500 \mathrm{~km} \mathrm{~s}^{-1}$. There is a marginal possibility 

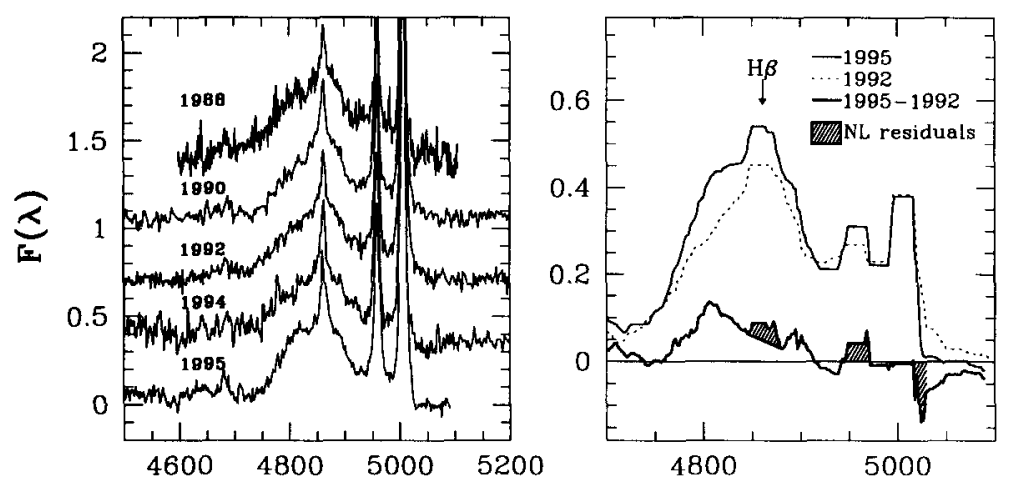

$\lambda(\AA)$

that a red peak also exists in the difference spectrum at $\sim 4890 \AA$. There may be a corresponding blueshifted component in the high-ionization line He II $\lambda 4686$ that arose at the same time.

Only a qualitative comparison of possible BLR emission models is feasible at this time. The significant profile displacement places a serious initial constraint on available models: (1) Accretion disk: The blueshift of the bulk profile as well as the difference spectrum (if double-peaked) appear to rule out simple disk models. (2) Binary black hole: The blueshifted bulk profile or variable component may represent one lower-mass black hole, but profile variations on the red side of $H \beta$, if any, suggest that the orbital eccentricity should be large, $e \geq 0.6$. (3) Radial or bicone models: several previous studies have pointed out how bicones illuminated by a central source or outflows of a more general kind should show variations that start in the blue wing.

We see some analogy to the variable displaced 'double peaks' that appeared in the past decade in Pictor A. The simplest interpretation for this variable feature views it as material entrained and accelerated in a biconical outflow that is closer to the central engine than the bulk of the broad-line emitting clouds. In the case of $4 \mathrm{C} 37.43$, the double-lobe structure provides evidence that the cone axis is aligned far from pole-on, suggesting that the actual ejection velocity could be much higher.

\section{References}

Boroson, T. A., \& Green, R.F. 1992, ApJS, 80, 109 (BG92).

Eracleous, M., \& Halpern, J. P. 1994, ApJS, 90, 1 (EH94).

Jackson, N., Penston, M. V., Pérez, E. 1991, MNRAS, 249, 577 (JPP91). Marziani, P., Sulentic, J.W., Dultzin-Hacyan, D., Calvani, M., \& Moles, M. 1996, ApJS, 104, 37 (M96). 